\title{
Assessment of suicide attempt and death in bipolar affective disorder: a combined clinical and genetic approach
}

\author{
Eric T. Monson ${ }^{1 凶}{ }^{凶}$, Andrey A. Shabalin ${ }^{1}$, Anna R. Docherty ${ }^{1}$, Emily DiBlasi ${ }^{1}$, Amanda V. Bakian (iD ${ }^{1,2}$, Qingqin S. Li (iD ${ }^{3}$, Douglas Gray ${ }^{1,4}$, \\ Brooks Keeshin $^{1,5}$, Sheila E. Crowell ${ }^{1,6,7}$, Niamh Mullins (iD) ${ }^{8,9}$, Virginia L. Willour ${ }^{10}$ and Hilary Coon (iD ${ }^{1,11,12}$
}

(c) The Author(s) 2021

\begin{abstract}
Bipolar disorder (BP) suicide death rates are 10-30 times greater than the general population, likely arising from environmental and genetic risk factors. Though suicidal behavior in BP has been investigated, studies have not addressed combined clinical and genetic factors specific to suicide death. To address this gap, a large, harmonized BP cohort was assessed to identify clinical risk factors for suicide death and attempt which then directed testing of underlying polygenic risks. 5901 individuals of European ancestry were assessed: 353 individuals with BP and 2498 without BP who died from suicide (BPS and NBPS, respectively) from a population-derived sample along with a volunteer-derived sample of 799 individuals with BP and a history of suicide attempt (BPSA), 824 individuals with BP and no prior attempts (BPNSA), and 1427 individuals without several common psychiatric illnesses per self-report (C). Clinical and subsequent directed genetic analyses utilized multivariable logistic models accounting for critical covariates and multiple testing. There was overrepresentation of diagnosis of PTSD (OR $=4.9,95 \% \mathrm{Cl}$ : $3.1-7.6)$ in $\mathrm{BPS}$ versus BPSA, driven by female subjects. PRS assessments showed elevations in BPS including PTSD $(\mathrm{OR}=1.3,95 \% \mathrm{Cl}: 1.1-1.5$, versus $\mathrm{C})$, femalederived $\mathrm{ADHD}(\mathrm{OR}=1.2,95 \% \mathrm{Cl}: 1.1-1.4$, versus $\mathrm{C}$ ), and male insomnia (OR=1.4, 95\%Cl: $1.1-1.7$, versus BPSA). The results provide support from genetic and clinical standpoints for dysregulated traumatic response particularly increasing risk of suicide death among individuals with BP of Northern European ancestry. Such findings may direct more aggressive treatment and prevention of trauma sequelae within at-risk bipolar individuals.
\end{abstract}

Translational Psychiatry (2021)11:379; https://doi.org/10.1038/s41398-021-01500-w

\section{INTRODUCTION}

Suicidal behavior, which can be defined in many ways, is here defined as behaviors that include suicide attempt and death by suicide [1]. Prior suicide attempt is consistently one of the strongest predictors of eventual death by suicide [2, 3]. However, the vast majority of individuals that attempt suicide will not die by suicide. Only $\sim 2.8 \%$ of individuals with at least one prior suicide attempt die by suicide [4]. Despite this, existing research on suicidal behavior primarily focuses on the evaluation of suicide attempt under the assumption that attempt acts as an adequate proxy for suicide death. Distinguishing factors important to suicide attempt versus suicide death will be crucial to the implementation of effective interventions to those most likely to die.

Patients with bipolar disorder (BP) have high rates of suicide attempt (30-50\%) and death (15-20\%) [5-7]. The rates for attempt and death are approximately twice those seen for major depression $[5,8]$ and the rate of death is greater than in any disorder except schizophrenia [9]. These features suggest potential elevation of biological risk of suicide specific to BP. For this study, we leveraged the largest cohort of population-ascertained suicide decedents available, representing over 7000 individuals collected over two decades in the state of Utah [10]. The majority of these subjects have genetic data available via array genotyping. Electronic health records (EHRs) of these subjects allowed for the identification of 353 individuals with BP who died by suicide. In addition to this unique sample, we utilized a large array-genotyped NIMH Genetics Initiative sample $(N=3050)$ including individuals with diagnosed BP, with and without a history of suicide attempt, and a comparison group that was screened for several common psychiatric illnesses via selfreport [11-14]. Together, these cohorts allowed a comprehensive study of clinical and hypothesis-driven genetic risk factors for suicide death in bipolar disorder and allowed for differentiation of risk factors between attempt and death.

\section{MATERIALS AND METHODS}

Sample selection

Two distinct sample sets were utilized. The first was composed of $>7000$ population-ascertained individuals who died from suicide from the Utah

\footnotetext{
${ }^{1}$ Department of Psychiatry \& Huntsman Mental Health Institute, University of Utah, Salt Lake City, UT, USA. ${ }^{2}$ Department of Family and Preventative Medicine, Division of Public Health, University of Utah, Salt Lake City, UT, USA. ${ }^{3}$ Neuroscience Therapeutic Area, Janssen Research and Development, Titusville, NJ, USA. ${ }^{4}$ VISN 19 Rocky Mountain MIRECC, George E. Wahlen VA Medical Center, Salt Lake City, UT, USA. ${ }^{5}$ Department of Pediatrics, University of Utah, Salt Lake City, UT, USA. ${ }^{6}$ Department of Psychology, University of Utah, Salt Lake City, UT, USA. ${ }^{7}$ Department of Obstetrics and Gynecology, University of Utah, Salt Lake City, UT, USA. ${ }^{8}$ Department of Psychiatry, Icahn School of Medicine at

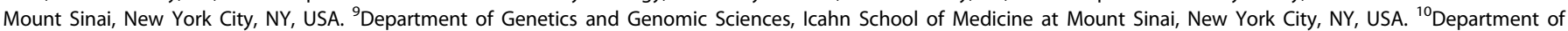

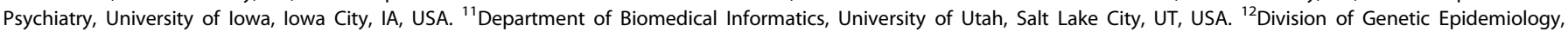
University of Utah, Salt Lake City, UT, USA. ${ }^{\bowtie}$ email: eric.monson@hsc.utah.edu
}

Received: 1 February 2021 Revised: 18 June 2021 Accepted: 23 June 2021

Published online: 07 July 2021 
Suicide Genetics Research Study (USGRS). These samples were collected through a collaboration with the Utah Medical Examiner's office and have been securely linked to electronic health record (EHR) information via the Utah Population Database (UPDB), a statewide data resource of demographic and health information (https://healthcare.utah.edu/huntsmancancerinstitute/ research/updb). Because the study design involved analysis of EHR followed by hypothesis-driven analyses of polygenic risk, a subset of 2851 suicide deaths with screened genome-wide genotyping data and who were linked to existing EHR data (see supplementary methods) were retained. Inpatient and ambulatory EHRs were obtained from Utah providers covering approximately $85 \%$ of the state, though they may not represent all health records for each individual. After EHR linking, identifying data were stripped before providing data to the research team; suicide cases were referenced by anonymous IDs. The individuals selected for this study had at least one prior diagnostic code for bipolar disorder (specifically bipolar I or bipolar NOS). EHR were also screened for schizophrenia diagnoses and these Individuals were removed from the BP group to increase the probability of diagnostic homogeneity. All individuals excluded from diagnosis in the BP suicide death group were also excluded from the non-BP suicide deaths to ensure that no known BP diagnoses (including diagnoses of cyclothymia, manic depressive disorder, and bipolar II) would be present within the non-BP suicide death group. A total of 353 individuals with BP who died from suicide (referred to as "BPS") and 2498 individuals without a diagnosis of BP who died from suicide (referred to as "NBPS") from Utah were included in the analyses. This sample represents the single largest known genotyped sample of BP suicide deaths and, in a post-hoc evaluation of power, was predicted to have $80 \%$ power to identify a clinical diagnostic difference between suicide death and comparison groups with an odds ratio of 1.7 as calculated via the UCSF online sample size calculator [15].

The second set of individuals was composed of a pre-existing deidentified genotyped dataset derived from the NIMH genetics initiative bipolar GWAS $[11-13,16]$ with a history of bipolar I or schizoaffective, bipolar type diagnoses as determined by formal evaluation and bestestimate diagnosis meeting criteria from the DSM-IIIR [17] or DSM-IV [18]. These individuals were selected for having complete clinical information from interview evaluation (individuals with missing information were excluded from this study). It is noted that interviews did not systematically evaluate for all included diagnoses, and that information from collected medical records and family informants that were used to support diagnoses were not available for all subjects (see Supplementary materials for more specific details of collected subject data). Individuals with a diagnosis of bipolar disorder and a history one or more suicide attempts $(N=799$, referred to as "BPSA") and individuals with a diagnosis of BP and no history of prior suicide attempt $(N=824$, referred to as "BPNSA") were selected for inclusion in the study with diagnostic and historical data being obtained via the Diagnostic Interview for Genetic Studies (DIGS v4) [19]. A comparison group of individuals who were screened for several common psychiatric disorders via self-report [14] were also included ( $N=1427$, referred to as " $C$ "). Briefly, screened illnesses excluded at the time of sample construction included major depression, psychosis, and bipolar disorder [12]. This sample has been described elsewhere (including acquisition and quality control efforts), noting that informed consent and appropriate IRB approval for all involved subjects was obtained in the original studies [11-14].

\section{Genotyping}

Utah suicide decedent DNAs were extracted from whole blood, and were genotyped using the Illumina Infinium PsychArray (https://www.illumina.com/ products/by-type/microarray-kits/infinium-psycharray.html) as described elsewhere [10]. DNAs from the NIMH BP and control populations were extracted from lymphoblastoid cell lines maintained at the NIMH DNA Repository (Infinite biologics, Rutgers RUCDR, https://www.rucdr.org/), and were genotyped using the Affymetrix Genome-Wide Human SNP Array 6.0 (https://www. thermofisher.com/us/en/home/life-science/microarray-analysis/affymetrix. $\mathrm{html}$ ) and processed as previously described $[12,16,20$,$] . Shared high-quality$ called variants from both platforms were combined and imputed via the Michigan Imputation Server [21] to a total of 7437997 high quality imputed variants. Extensive quality control steps, including assessment for ancestry and relatedness, were utilized to prepare this sample for analysis (see Supplementary methods). Due to sensitivity of polygenic risk scores to ancestry effects, this study focused only on individuals of $>90 \%$ European ancestry.

\section{Analysis of sample characteristics}

Statistical evaluation of the distribution of sex, age, education level, and clinical categories across all comparison groups were evaluated by chisquare (sex, education, clinical categories) or ANOVA (age).

\section{Analyses of clinical data}

Five clinical diagnostic categories were constructed from available diagnoses based on consensus of M.D./Ph.D.-level clinicians (E.M., B.K., A. D.) for all subjects with full details within the Supplementary methods and Supplementary Table S1. Briefly, these categories represented nontraumatic anxiety disorders, behavioral disorders, personality disorders, eating disorders, and PTSD. The primary clinical analysis compared BPS, BPSA, and BPNSA within these categories.

All clinical categories were also secondarily evaluated to determine if observed effects were specific to BPS. NBPS were compared with BPS for these assessments.

All clinical analyses utilized logistic multivariable regression in $R$ [22] accounting for age, sex, and education level. All variables were derived from single, independent measures for each subject. It was also noted that BPS had considerably more clinical diagnoses, on average, than NBPS, necessitating the inclusion of a clinical diagnosis count covariate within analyses comparing these groups. Effect size estimates were calculated via adjusted odds ratio from each model. Correction for multiple testing of 15 primary and 30 sex-specific clinical analyses utilized the Benjamini-Hochberg method with a false discovery rate of 0.05 .

\section{Analyses of genetic data}

PRS calculations of several phenotypes, selected via significant clinical analysis results, were generated from 9 GWAS datasets (19 PRS total). Summary GWAS data arose from meta-analyses with publicly available summary statistics for ADHD [23,24], anxiety [25], insomnia [26], PTSD [27], suicide attempt [28], and neuroticism [29]. Many of these sets included summary statistics for population subsets, including male- and female-only analyses, referred to here as "sex-derived" sets, which were also analyzed. Suicide death PRS was calculated from the USGRS suicide death GWAS using a cross-validation approach described elsewhere [10]. Suicide attempt in bipolar disorder PRS were calculated from published Psychiatric Genomic Consortium data [28] with all overlapping study subjects removed. PRS calculations were conducted using PRSice 2.0 [30] with a $p$-value threshold of 1.0 as described in the Supplemental note. All PRS were standardized to $Z$-scores prior to statistical analysis.

Pairwise comparisons of BPS, BPSA, BPNSA, and C utilized multivariable logistic regression models in R [22], accounting for age, sex, and the first 10 principal components to control for residual ancestry effects. As with the clinical variables and covariates, all variables were obtained from independent measures without duplication. PRS measures were evaluated to have similar variance across groups during assessment and as visualized in plots. Effect size estimates were calculated by adjusted odds ratio from each model. Correction for multiple testing of 114 primary and 204 sexspecific PRS analyses utilized Benjamini-Hochberg calculations with a false discovery rate of 0.05 .

\section{RESULTS}

\section{Sample evaluation}

Sample demographics, including frequency of comorbid diagnoses within the defined clinical categories and statistical evaluation of the distribution across the groups for each demographic are outlined in Table 1. It is noted that the groups varied from one another significantly, but particularly striking differences can be appreciated in the sex distribution of each group. These differences are consistent with expectations that more males than females die from suicide, and more females than males attempt suicide [31]. However, it is notable that the excess of male deaths was significantly lower in BPS when compared to NBPS $(62.0 \%$ of BPS being male versus $79.5 \%$ of NBPS being male, $\left.\mathrm{OR}=0.42,95 \% \mathrm{Cl}=0.33-0.53 ; X^{2}=54.1, P=1.9 \times 10^{-13}\right)$.

\section{Clinical analyses of BPS, BPSA, and BPNSA}

Complete results can be viewed within Supplementary Tables S2 and S3 with odds ratios and confidence intervals displayed in 
Table 1. Study sample demographics by comparison group.

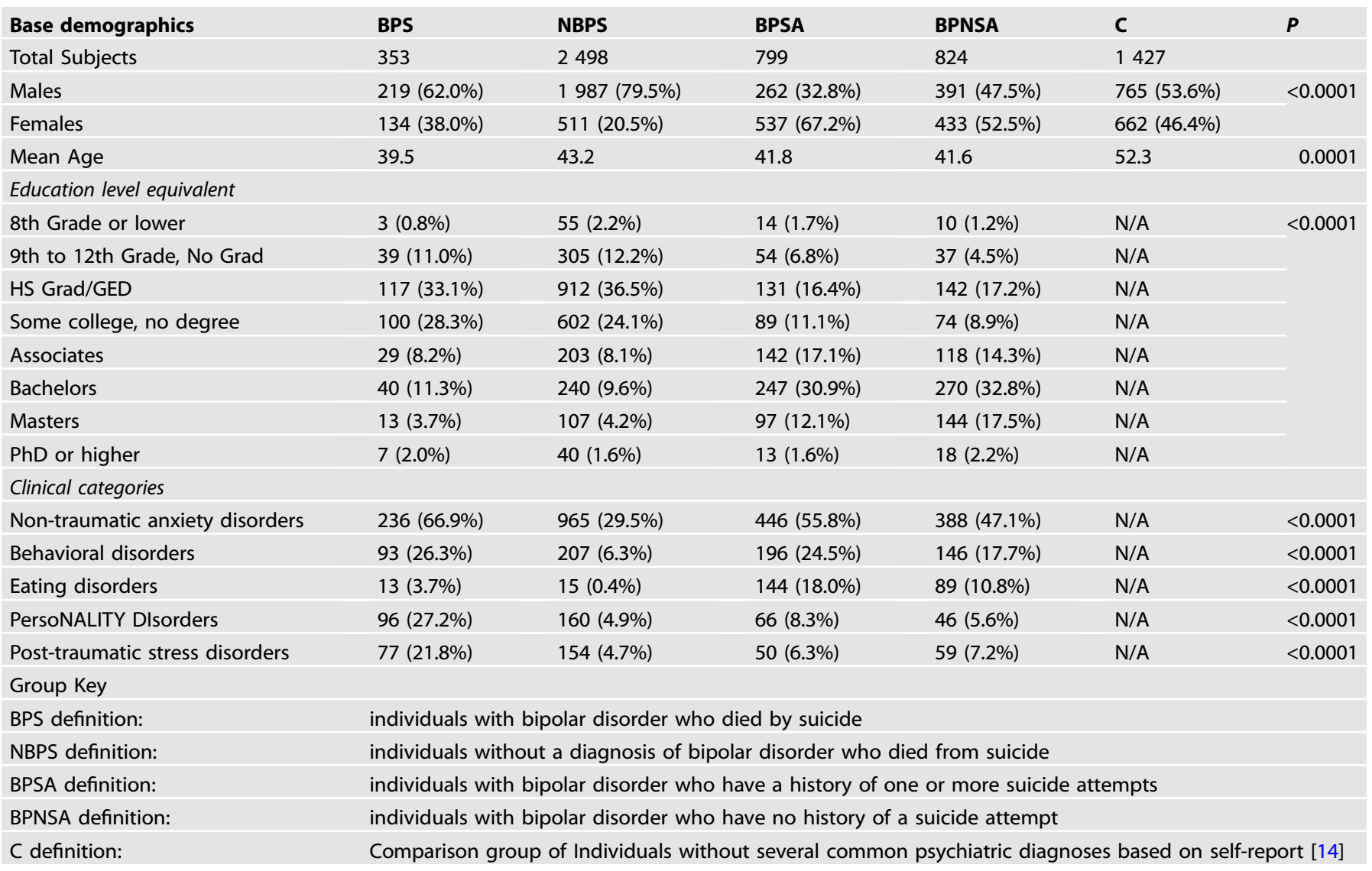

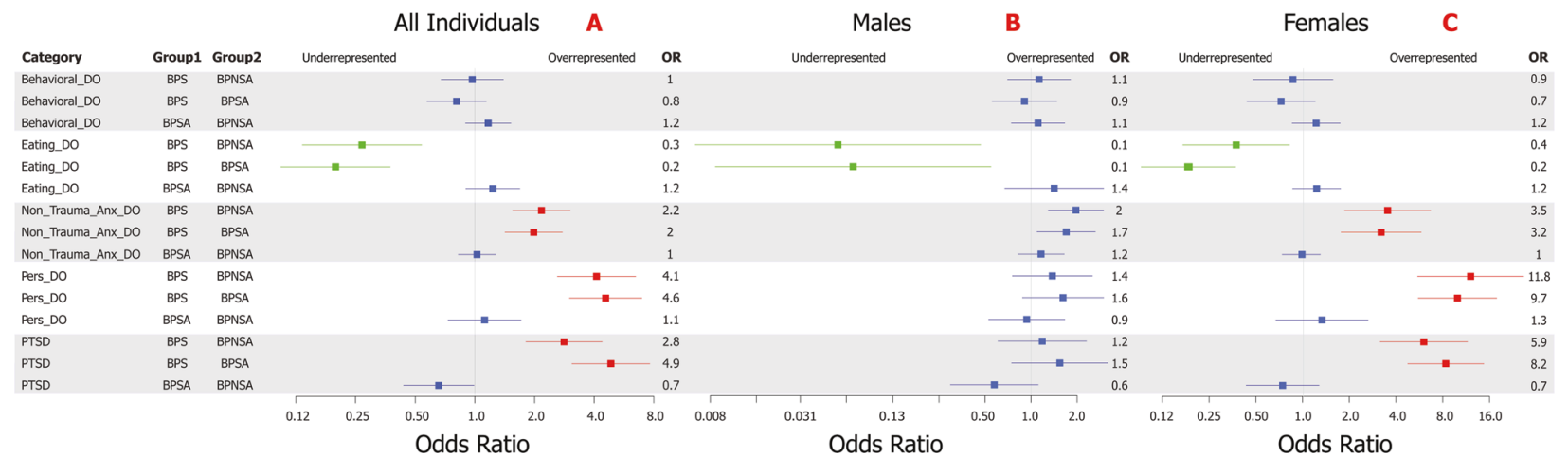

Fig. 1 BPS versus BPSA and BPNSA clinical category analysis results. Forest plot distribution of corrected odds ratios of the primary clinical category comparisons (with 95\% confidence interval represented by whiskers) within all individuals (A), males (B), and females (C). Labeling of comparison groups is as follows: BPS = individuals with bipolar disorder who died by suicide, BPSA = individuals with bipolar disorder who have a history of one or more suicide attempts, and BPNSA = individuals with bipolar disorder who have no history of a suicide attempt. Significant results are colored with overrepresentation shown in red and underrepresentation in green. Non-significant results are shown in blue. Results were corrected for multiple testing via the Benjamini-Hochberg method with an FDR of 0.05 for a total of 15 tests in the primary analysis (A) and 30 in the sex-specific analyses (B, C) and for critical covariates.

Fig. 1. All results are corrected for multiple testing and covariates. BPS versus BPSA showed overrepresentation for diagnoses of PTSD $\left(\mathrm{OR}=4.9, \quad 95 \% \mathrm{Cl}=3.1-7.6 ; \quad P=6.0 \times 10^{-11}\right)$, personality disorders $\left(\mathrm{OR}=4.6,95 \% \mathrm{Cl}=3.0-7.0 ; P=2.2 \times 10^{-11}\right.$; noting the caveat discussed in the limitations), and non-traumatic anxiety disorders $\left(\mathrm{OR}=2.0,95 \% \mathrm{Cl}=1.4-2.8 ; P=1.3 \times 10^{-4}\right)$. Eating disorder diagnoses were significantly reduced within BPS versus BPSA $\left(\mathrm{OR}=0.2,95 \% \mathrm{Cl}=0.1-0.4 ; P=2.2 \times 10^{-6}\right)$. No comparisons were significant between BPSA and BPNSA.
Secondary sex-specific results are shown in Fig. $1 \mathrm{~B}$ and $\mathrm{C}$. Females strongly drove overrepresentations of $\mathrm{PTSD}(\mathrm{OR}=8.2$, $\left.95 \% \mathrm{Cl}=4.7-14.4 ; P=3.4 \times 10^{-11}\right)$, personality disorder $(\mathrm{OR}=9.7$, $\left.95 \% \mathrm{Cl}=5.5-17.4 ; \quad P=4.5 \times 10^{-13}\right)$, and non-traumatic anxiety disorder $\left(\mathrm{OR}=3.2,95 \% \mathrm{Cl}=1.7-5.7 ; P=6.1 \times 10^{-4}\right)$ in $\mathrm{BPS}$ versus BPSA. Females also drove the reduced rate of eating disorder diagnoses in BPS versus BPSA $(\mathrm{OR}=0.2,95 \% \mathrm{Cl}=0.1-0.4 ; P=$ $\left.1.1 \times 10^{-5}\right)$. Though male BPS versus male BPSA showed nominal overrepresentation of non-traumatic anxiety disorders, personality 


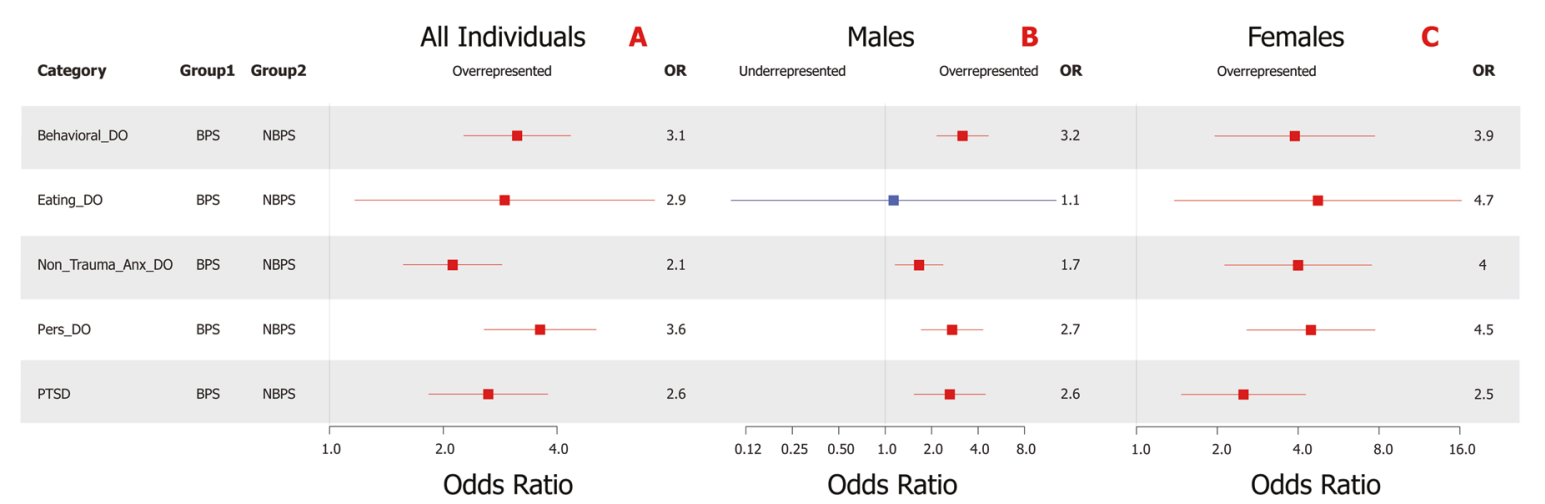

Fig. 2 BPS versus NBPS clinical category analysis results. Forest plot distribution of corrected odds ratios of the suicide-only clinical category comparisons (with 95\% confidence interval represented by whiskers) within all subjects (A), male subjects (B), and female subjects (C). Labeling of comparison groups is as follows: BPS = individuals with bipolar disorder who died by suicide and NBPS = individuals without a diagnosis of bipolar disorder who died from suicide. Significant results are colored with overrepresentation shown in red and underrepresentation in green. Non-significant results are shown in blue. Results were corrected for multiple testing via the Benjamini-Hochberg method with an FDR of 0.05 for a total of 5 tests in the primary analysis (A) and 10 in the sex-specific analyses (B, C) and for critical covariates.

disorders, and PTSD, none of these results survived correction for multiple testing.

\section{Clinical analysis of BPS versus NBPS}

Comparisons are shown in Fig. 2 with complete results in Supplementary Tables S4 and S5. Even after correction for medical record completeness and years of education, BPS were elevated versus NBPS for all comorbid psychiatric diagnoses, including within sex-specific analyses (except for the male eating disorders comparison). The strongest elevations were noted within personality disorders $\left(\mathrm{OR}=3.6,95 \% \mathrm{Cl}=2.6-5.1 ; P=9.1 \times 10^{-13}\right)$ and behavioral disorders $\left(\mathrm{OR}=3.1,95 \% \mathrm{Cl}=2.3-4.3 ; P=1.3 \times 10^{-11}\right)$. In addition, findings show similar effect sizes within the sexspecific comparisons (Fig. 2B and C), regardless of sex, though all diagnoses were seen at higher frequencies within females, both within the BPS and NBPS.

\section{Genetic risk analysis across BPS, BPSA, BPNSA, and C}

See Figs. 3 and 4 with full results in Supplementary Tables S6 and S7.

i. Suicide attempt in MDD and BP and suicide death PRS.

Suicide death PRS (Fig. 3A) was elevated in BPS versus BPSA $\left(\mathrm{OR}=1.6,95 \% \mathrm{Cl}=1.4-1.9 ; P=7.8 \times 10^{-10}\right)$. Suicide attempt in BP PRS (Fig. 3B) showed elevations in BPS versus BPNSA $\left(\mathrm{OR}=1.5,95 \% \mathrm{Cl}=1.3-1.8 ; P=1.9 \times 10^{-6}\right)$ and BPSA versus $B P N S A\left(O R=1.5,95 \% C l=1.3-1.6 ; P=1.1 \times 10^{-11}\right)$. Suicide attempt in BP was also reduced in BPNSA versus $C$ $\left(\mathrm{OR}=0.7,95 \% \mathrm{Cl}=0.7-0.8 ; P=4.2 \times 10^{-8}\right)$. PRS for suicide attempt within major depressive disorder (Fig. 3C) was elevated in BPS compared with all comparison groups, including versus $\mathrm{BPSA}(\mathrm{OR}=1.3,95 \% \mathrm{Cl}=1.1-1.6 ; P=1.8 \times$ $10^{-2}$ ).

ii. Anxiety, and neuroticism PRS.

Anxiety (Fig. 3D; $\mathrm{OR}=1.2,95 \% \mathrm{Cl}=1.1-1.3, P=1.8 \times$ $10^{-2}$ ) and neuroticism (worry subcluster, not shown; OR = $1.2,95 \% \mathrm{Cl}=1.1-1.3, P=6.0 \times 10^{-3}$ ) showed elevated PRS in BPSA versus $C$. Notably, PRS for anxiety and neuroticism also showed either significant or nominal elevation in BPS, BPSA, and BPNSA versus $C$, and ad-hoc comparisons of BPS, BPSA, and BPNSA combined into a single comparison group versus $C$ showed $P=5.6 \times 10^{-5}$ for anxiety and $3.8 \times 10^{-4}$ for neuroticism.

iii. PTSD and behavioral PRS.

The PTSD GWAS published summary statistics for males, females, and all subjects [27]. All-subject (not shown;
$\mathrm{OR}=1.3, \quad 95 \% \mathrm{Cl}=1.1-1.5, \quad P=7.8 \times 10^{-3}$ ) and malederived PTSD PRS (Fig. $4 \mathrm{~A}$; $O R=1.3,95 \% \mathrm{Cl}=1.1-1.5$, $P=8.0 \times 10^{-3}$ ) were elevated within BPS versus $C$. Femalederived PTSD PRS (Fig. 4B), however, was elevated in both $B P S A$ versus $C\left(O R=1.2,95 \% C l=1.1-1.3, P=3.6 \times 10^{-3}\right)$ and $B P S$ versus $C\left(O R=1.2,95 \% C l=1.1-1.4, P=2.5 \times 10^{-2}\right)$. None of these comparisons remained significant in sexspecific analyses, but effect sizes were similar.

Female-derived ADHD PRS (Fig. 4C) was elevated within $\mathrm{BPS}$ versus $\mathrm{C}\left(\mathrm{OR}=1.2,95 \% \mathrm{Cl}=1.1-1.4, P=2.0 \times 10^{-2}\right)$.

iv. Sex-specific PRS.

Sex-specific PRS analyses (Supplementary Table S7) generally reproduced findings with similar effect sizes in both sexes, but often did not survive correction for multiple testing in the setting of smaller comparison groups. One new finding was identified, however: male-specific polygenic risk for insomnia (daytime napping subgroup, Fig. 4D) was elevated in BPS versus BPSA with $\mathrm{OR}=1.4,95 \% \mathrm{Cl}=$ $1.1-1.7, P=4.3 \times 10^{-2}$.

v. BPS versus NBPS PRS.

PRS analyses of BPS versus NBPS (Supplementary Tables S8-S9) showed no significant differences between groups, including within sex-specific analyses.

\section{DISCUSSION}

Suicide attempt is often used as a proxy for suicide death and, as such, frequently serves as the primary phenotype within studies of suicide. Suicide attempts and deaths, however, are separate groups that overlap. The unique resource of the USGRS dataset allowed more thorough exploration of suicide death within BP, identifying several potentially important clinical and genetic associations that may aid in identifying and differentiating those at highest risk for suicide attempt and death.

\section{The role of trauma and its enduring effects in suicidal behavior}

This study is the first, to our knowledge, to identify combined clinical and genetic evidence of factors that may distinguish risk for suicide death from attempt in BP. Specifically, PTSD and personality disorder diagnoses were strongly elevated in BPS versus BPSA. In addition, clinically informed genetic analyses identified elevated polygenic risk for PTSD in BPS. Trauma, and subsequent response, is a common factor in these findings. A 

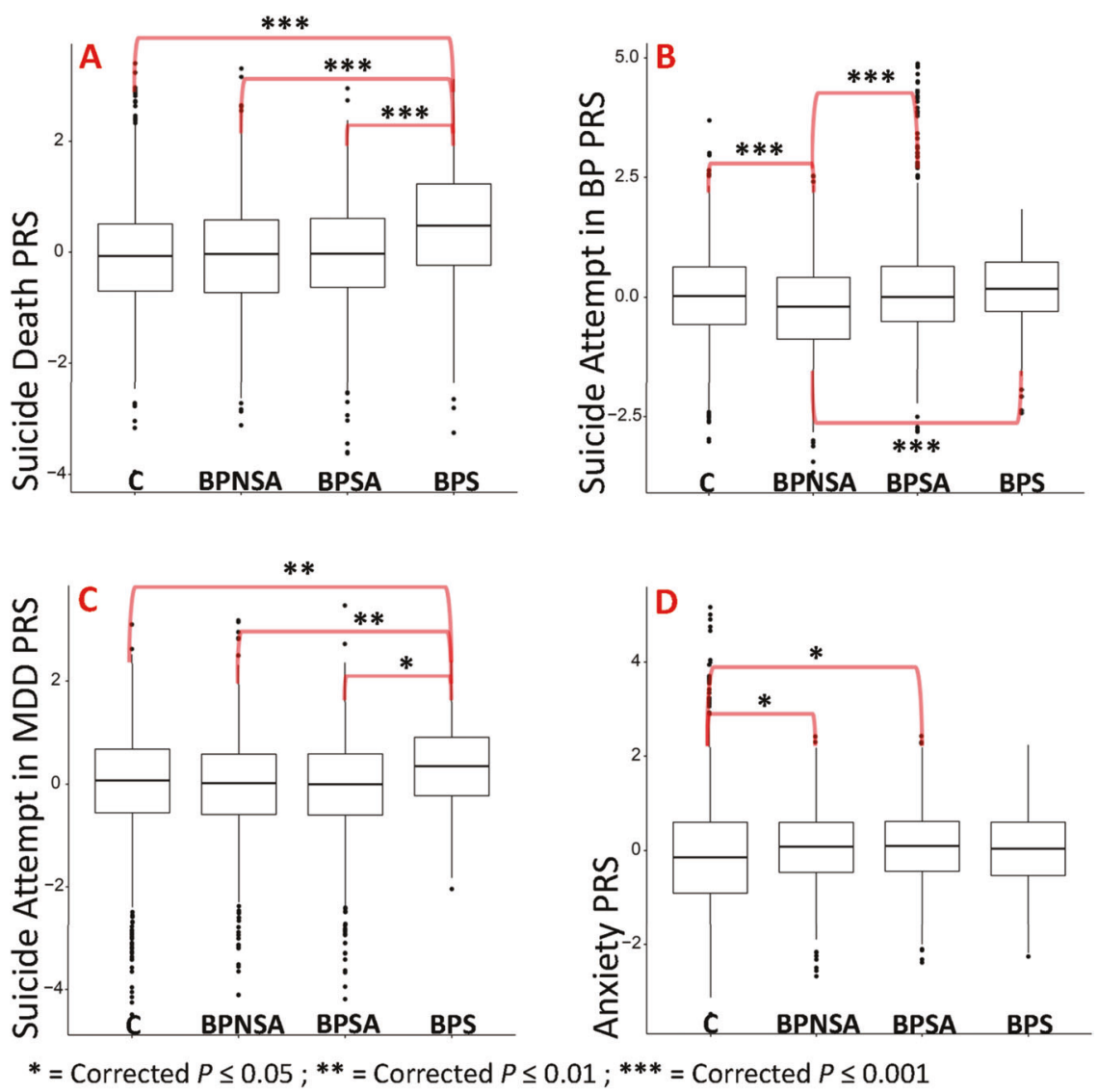

Fig. 3 Suicide death, attempt, and anxiety PRS results. Box plot representations of the top findings from polygenic risk score association testing. Each plot represents comparison group ( $x$-axis) versus standardized polygenic risk score for the given phenotype ( $y$-axis). A Suicide death PRS. B Suicide attempt in bipolar disorder PRS. C Suicide attempt in MDD PRS. D Anxiety PRS. Comparison group definitions: BPS = individuals with bipolar disorder who died by suicide, BPSA = individuals with bipolar disorder who have a history of one or more suicide attempts, BPNSA = individuals with bipolar disorder who have no history of a suicide attempt, and $\mathrm{C}=$ comparison individuals without several common psychiatric illnesses per self-report [14]. Selected results shown; all displayed results have been corrected for multiple testing (Benjamini-Hochberg method with FDR of 0.05 correcting for 114 tests for all displayed results) and account for critical covariates. All shown results arose from evaluating all subjects (male and female).

history of trauma is required for PTSD [32] and is correlated with a more severe course [33] with an increased risk for suicidal behavior in BP $[34,35]$. Trauma is also frequently present in personality disorders [32] which are associated with suicidal behavior [36] and often comorbid with PTSD [37].

Such findings may also provide unifying support for the role of stress response pathways such as the hypothalamic pituitary axis (HPA) [38]. Prior evidence suggests that genetic disruption of the HPA-axis may interact with trauma/severe stress exposure to increase risk for suicide attempt [39]. This study provides novel evidence that traumatic disruption may increase risk of death from suicide. Indeed, recent evidence has arisen that early-life traumatic exposure in the setting of elevated polygenic risk for BP is significantly correlated with an increase in suicide attempts [40]. Taken together, the clinical and genetic findings of this study support the long-standing stress-diathesis model for suicidal behavior in BP [41], and specifically extend those findings to risk for suicide death.

Data from this study also demonstrate potentially important sex-related differences, with trauma-related diagnoses being driven by female BPS. In contrast, males and female BPS demonstrate relatively equal effects within polygenic risk for PTSD. This suggests potential differences in care seeking or clinical presentation in males that may not lead to the same diagnoses. BPS also showed elevated male- and female-derived PTSD PRS, but BPSA was only significantly associated with the female- derived PTSD PRS. This may indicate that genetic loci that interact with certain types of trauma, such as military trauma exposures identified within males of the PTSD GWAS [27], may be more closely associated with suicide death risk than attempt.

Finally, the clinical evaluation of BPS versus NBPS showed a striking overrepresentation of comorbid diagnoses, particularly trauma-associated diagnoses, but polygenic risk comparison yielded no significant findings. It is possible that genetic liability among BPS and NBPS is similar, but patients diagnosed with BP may receive additional clinical evaluation leading to identification of comorbid diagnoses such as PTSD.

\section{Other potential risk factors}

The clinically directed genetic analyses generated novel correlations of ADHD and insomnia polygenic risk in BPS versus C. ADHD diagnosis was also overrepresented in BPS versus NBPS. ADHD has been shown to be correlated with suicidal behavior [42] and may increase risk when comorbid with BP [43]. Together, ADHD and BP could be theorized to increase risk for "impulsive aggression", a potentially important risk factor for suicidal behavior [44]. Insomnia has also been correlated with increased suicide behavior risk [45] and may be an important predictive factor for the presence of comorbid disease, such as PTSD [46]. It is notable that in comparing BPS to NBPS, $44.8 \%$ versus $18.3 \%$ of females and $28.4 \%$ versus $13.2 \%$ of males had a concurrent diagnosis of insomnia, respectively. This suggests that female BPS are more 

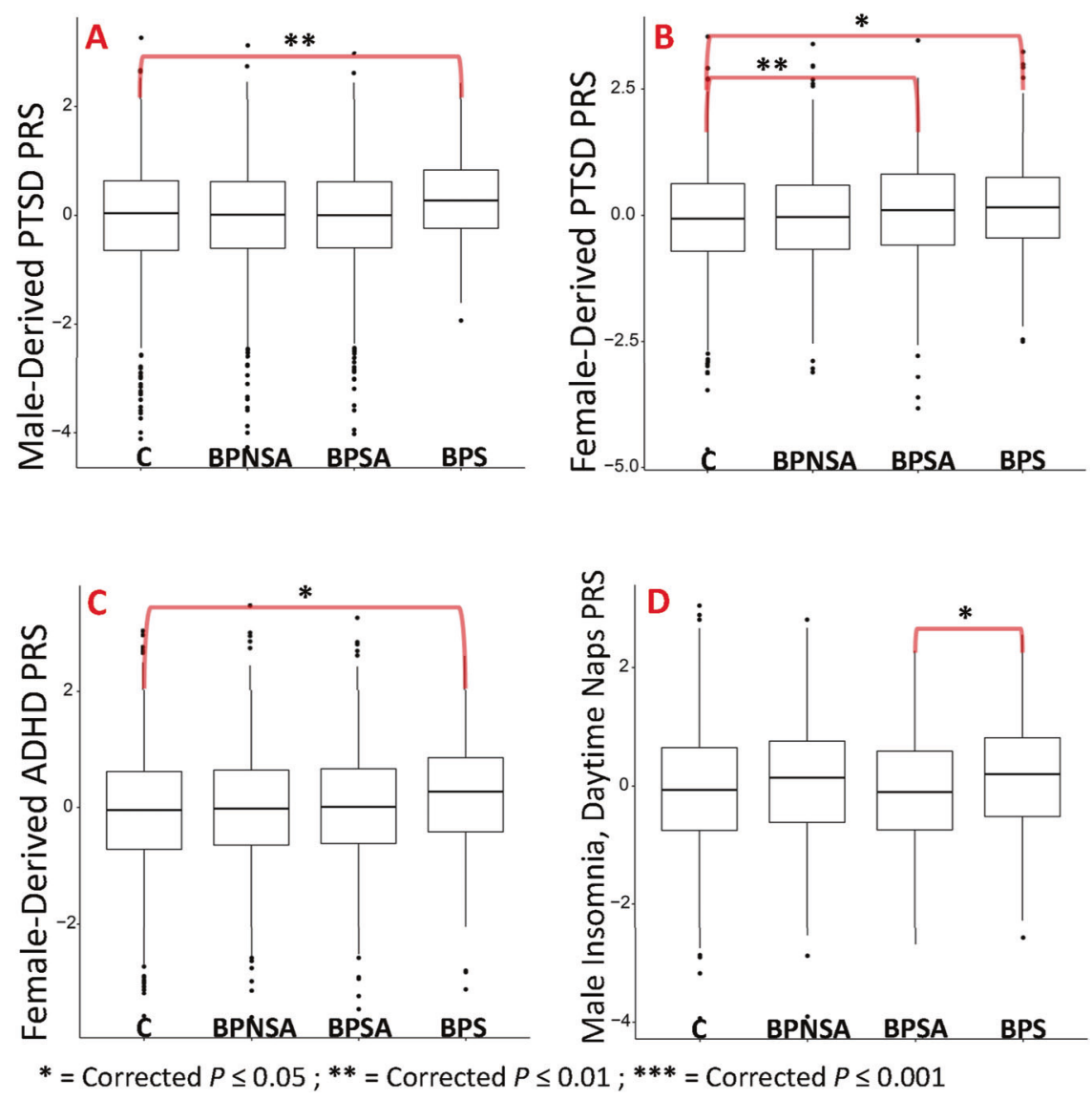

Fig. 4 PTSD and behavioral PRS results. Box plot representations of the top findings from polygenic risk score association testing. Each plot represents comparison group ( $x$-axis) versus standardized polygenic risk score for the given phenotype ( $y$-axis). A Male-derived PTSD PRS. B Female-derived PTSD PRS. C Female-derived ADHD PRS. D Male only (sex-specific) insomnia (daytime napping subgroup) PRS. Comparison group definitions: BPS = individuals with bipolar disorder who died by suicide, BPSA = individuals with bipolar disorder who have a history of one or more suicide attempts, BPNSA = individuals with bipolar disorder who have no history of a suicide attempt, and C $=$ comparison individuals without several common psychiatric illnesses per self-report [14]. Selected results shown; all displayed results have been corrected for multiple testing (Benjamini-Hochberg method with FDR of 0.05 correcting for 114 tests for A-C and 204 tests for D) and account for critical covariates. Note that sex-derived refers to PRS calculated based on weighted results from the given sex in the original GWAS. All results were evaluated from all (male and female) subjects in the current study with the exception of $\mathbf{D}$, which was an evaluation of only males.

frequently diagnosed with insomnia despite a male-driven genetic finding, which may indicate sex-specific differences in diagnosis or care seeking.

\section{Limitations}

This study had several limitations. Among these is modest sample size, though the assessed sample of BPS is the largest known sample of its kind. Also, replication is currently not possible as no comparable BPS sample is currently known to exist. Though not the focus of this study, efforts are also underway to collect larger BPSA and BPNSA samples with clinical data to allow more effective comparisons of these groups.

The use of two distinct cohorts introduced several potential limitations. Different genotyping arrays led to a limited number of overlapping variants, somewhat limiting the efficacy of imputation and PRS calculation. In addition, population ascertainment differed substantially: a general population sample (USGRS) versus an assembled research sample (NIMH), both with strengths but potentially biased comparisons. For example, the USGRS samples were not evaluated with a comprehensive diagnostic interview, potentially missing important comorbidities and weakening current associations. It must also be noted that diagnoses within a population sample arise only through individuals seeking clinical encounters and are less likely to represent every diagnosis an individual might have. This leads to a high likelihood that individuals with undiagnosed BP may be present within NBPS, potentially weakening comparisons between these groups. Conversely, the NIMH sample represents voluntary cohorts that may not adequately reflect the general population, but who were rigorously assessed by multiple providers via a consistent, extensive questionnaire to provide best estimate diagnoses. Despite this rigorous evaluation, however, all potentially relevant comorbidities, and particularly the personality disorders other than antisocial personality disorder, were not systematically evaluated as part of the core questionnaire, being identified through family informant, medical records, and early life trauma evaluation which were not available for all subjects. Indeed, it was noted that $<1 \%$ of BP individuals within the NIMH cohort were diagnosed with borderline personality disorder, though a recent meta-analysis of the frequency of comorbid borderline personality disorder in bipolar disorder predicted an average of 21.6\% [47], suggesting that many diagnoses may have been missed in this cohort. Finally, the evaluation of only Northern European subjects limits the generalizability of this study and was necessitated by a limited number of samples from other ethnicities. Ongoing efforts to collect a larger, more diverse, and cohesive sample are underway. Despite these inherent challenges, however, it is notable that the comparison of such datasets is necessitated by the relative rarity of these phenotypes (particularly suicide death) and is supported by evidence of a convergent finding within 
clinical and genetic data evaluations of prior trauma as a potential factor in suicide death risk in BP, illustrating the potential power of this complimentary approach.

\section{CONCLUSION}

This study represents the first large-scale evaluation of suicide death in BP to utilize a combined clinical and genetic approach. In identifying converging evidence of factors specifically associated with suicide death, particularly prior trauma and its associated phenotypes, this study provides potentially tractable targets for future evaluation and indicates the need to specifically collect and evaluate individuals who have died from suicide to best characterize risk factors for this preventable outcome. Findings may serve to improve current screening measures for suicide death risk and, ultimately, help reduce death by suicide.

\section{REFERENCES}

1. Turecki G, Brent DA, Gunnell D, O'Connor RC, Oquendo MA, Pirkis J, et al. Suicide and suicide risk. Nat Rev Dis Primers. 2019;5:74. https://doi.org/10.1038/s41572019-0121-0.

2. Owens $D$, Horrocks J, House A. Fatal and non-fatal repetition of self-harm. Systematic review. $\mathrm{Br} J$ Psychiatry. 2002;181:193-9. https://doi.org/10.1192/ bjp.181.3.193.

3. Bostwick JM, Pabbati C, Geske JR, McKean AJ. Suicide attempt as a risk factor for completed suicide: even more lethal than we knew. Am J Psychiatry. 2016;173:1094-100. https://doi.org/10.1176/appi.ajp.2016.15070854.

4. Hawton K, Fagg J. Suicide, and other causes of death, following attempted suicide. Br J Psychiatry. 1988;152:359-66. https://doi.org/10.1192/bjp.152.3.359.

5. Valtonen $H$, Suominen $K$, Mantere $O$, Leppämäki $S$, Arvilommi $P$, Isometsä $E T$. Suicidal ideation and attempts in bipolar I and II disorders. J Clin Psychiatry. 2005;66:1456-62.

6. Gonda X, Pompili M, Serafini G, Montebovi F, Campi S, Dome P, et al. Suicidal behavior in bipolar disorder: epidemiology, characteristics and major risk factors. J Affect Disord. 2012;143:16-26. https://doi.org/10.1016/j.jad.2012.04.041.

7. Miller JN, Black DW. Bipolar disorder and suicide: a review. Curr Psychiatry Rep. 2020;22:6. https://doi.org/10.1007/s11920-020-1130-0.

8. Chen YW, Dilsaver SC. Lifetime rates of suicide attempts among subjects with bipolar and unipolar disorders relative to subjects with other Axis I disorders. Biol. psychiatry. 1996;39:896-9. https://doi.org/10.1016/0006-3223(95)00295-2.

9. Yeh HH, Westphal J, Hu Y, Peterson EL, Williams LK, Prabhakar D, et al. Diagnosed mental health conditions and risk of suicide mortality. Psychiatr. Serv. 2019;70:750-7. https://doi.org/10.1176/appi.ps.201800346.

10. Docherty AR, Shabalin AA, DiBlasi E, Monson E, Mullins N, Adkins DE, et al. Genome-wide association study of suicide death and polygenic prediction of clinical antecedents. Am J Psychiatry. 2020;177:917-27. https://doi.org/10.1176/ appi.ajp.2020.19101025.

11. Genomic survey of bipolar illness in the NIMH genetics initiative pedigrees: a preliminary report. Am J Med. Genet. 1997;74:227-37.

12. Smith EN, Bloss CS, Badner JA, Barrett T, Belmonte PL, Berrettini W, et al. Genomewide association study of bipolar disorder in European American and African American individuals. Mol Psychiatry. 2009;14:755-63. https://doi.org/10.1038/ mp.2009.43.

13. McMahon FJ, Akula N, Schulze TG, Muglia P, Tozzi F, Detera-Wadleigh SD, et al. Meta-analysis of genome-wide association data identifies a risk locus for major mood disorders on 3p21.1. Nat Genet. 2010;42:128-31. https://doi.org/10.1038/ ng.523.

14. Sanders AR, Levinson DF, Duan J, Dennis JM, Li R, Kendler KS, et al. The Internetbased MGS2 control sample: self report of mental illness. Am. J. psychiatry. 2010;167:854-65. https://doi.org/10.1176/appi.ajp.2010.09071050.

15. Kohn MA, S. J. Sample Size Calculators, 2021, https://sample-size.net/sample-sizeproportions/.

16. Willour VL, Seifuddin F, Mahon PB, Jancic D, Pirooznia M, Steele J, et al. A genome-wide association study of attempted suicide. Mol Psychiatry. 2012;17:433-44. https://doi.org/10.1038/mp.2011.4.

17. American Psychiatric Association. Desk reference to the diagnostic criteria from DSM-III. American Psychiatric Association; 1982.

18. American Psychiatric Association. Diagnostic criteria from DSM-IV. The Association; 1994.

19. Nurnberger JI Jr, Blehar MC, Kaufmann CA, York-Cooler C, Simpson SG, HarkavyFriedman J, et al. Diagnostic interview for genetic studies. Rationale, unique features, and training. NIMH Genetics Initiative. Arch Gen Psychiatry. 1994:51:849-59. discussion 863-844.

20. Smith EN, Koller DL, Panganiban C, Szelinger S, Zhang P, Badner JA, et al. Genome-wide association of bipolar disorder suggests an enrichment of replicable associations in regions near genes. PLoS Genet. 2011;7:e1002134. https:// doi.org/10.1371/journal.pgen.1002134.

21. Das $S$, Forer $L$, Schönherr $S$, Sidore $C$, Locke $A E$, Kwong $A$, et al. Next-generation genotype imputation service and methods. Nat Genet. 2016;48:1284-7. https:// doi.org/10.1038/ng.3656.

22. R: A Language and Environment for Statisitcal Computing ( $R$ Foundation for Statistical Computing, Vienna, Austria, 2020).

23. Martin J, Walters RK, Demontis D, Mattheisen M, Lee SH, Robinson E, et al. A genetic investigation of sex bias in the prevalence of attention-deficit/hyperactivity disorder. Biol Psychiatry. 2018;83:1044-53. https://doi.org/10.1016/j. biopsych.2017.11.026.

24. Demontis D, Walters RK, Martin J, Mattheisen M, Als TD, Agerbo E, et al. Discovery of the first genome-wide significant risk loci for attention deficit/hyperactivity disorder. Nat Genet. 2019;51:63-75. https://doi.org/10.1038/s41588-018-0269-7.

25. Otowa T, Hek K, Lee M, Byrne EM, Mirza SS, Nivard MG, et al. Meta-analysis of genome-wide association studies of anxiety disorders. Mol. Psychiatry. 2016;21:1391-9. https://doi.org/10.1038/mp.2015.197.

26. Jansen PR, Watanabe K, Stringer S, Skene N, Bryois J, Hammerschlag AR, et al. Genome-wide analysis of insomnia in 1,331,010 individuals identifies new risk loci and functional pathways. Nat. Genet. 2019;51:394-403. https://doi.org/ 10.1038/s41588-018-0333-3.

27. Nievergelt $C M$, Maihofer $A X$, Klengel T, Atkinson EG, Chen CY, Choi KW, et al. International meta-analysis of PTSD genome-wide association studies identifies sex- and ancestry-specific genetic risk loci. Nat Commun. 2019;10:4558. https:// doi.org/10.1038/s41467-019-12576-w.

28. Mullins N, Bigdeli TB, Børglum AD, Coleman J, Demontis D, Mehta D, et al. GWAS of suicide attempt in psychiatric disorders and association with major depression polygenic risk scores. Am J Psychiatry. 2019;176:651-60. https://doi.org/10.1176/ appi.ajp.2019.18080957.

29. Nagel M, Jansen PR, Stringer S, Watanabe K, de Leeuw CA, Bryois J, et al. Metaanalysis of genome-wide association studies for neuroticism in 449,484 individuals identifies novel genetic loci and pathways. Nat Genet. 2018;50:920-7. https://doi.org/10.1038/s41588-018-0151-7.

30. Euesden J, Lewis CM, O'Reilly PF. PRSice: polygenic risk score software. Bioinformatics. 2015;31:1466-8. https://doi.org/10.1093/bioinformatics/btu848.

31. Schrijvers DL, Bollen J, Sabbe BG. The gender paradox in suicidal behavior and its impact on the suicidal process. J Affect Disord. 2012;138:19-26. https://doi.org/ 10.1016/j.jad.2011.03.050.

32. American Psychiatric Association. \& American Psychiatric Association. DSM-5 Task Force. Diagnostic and statistical manual of mental disorders: DSM-5. 5th edn, American Psychiatric Association, 2013.

33. Etain B, Aas M, Andreassen OA, Lorentzen S, Dieset I, Gard S, et al. Childhood trauma is associated with severe clinical characteristics of bipolar disorders. J Clin Psychiatry. 2013;74:991-8. https://doi.org/10.4088/JCP.13m08353.

34. Schaffer A, Isometsä ET, Azorin JM, Cassidy F, Goldstein T, Rihmer Z, et al. A review of factors associated with greater likelihood of suicide attempts and suicide deaths in bipolar disorder: Part II of a report of the International Society for Bipolar Disorders Task Force on Suicide in Bipolar Disorder. Aust N Z J Psychiatry. 2015;49:1006-20. https://doi.org/10.1177/0004867415594428.

35. Park, YM, Shekhtman, T, Kelsoe, JR. Effect of the Type and Number of Adverse Childhood Experiences and the Timing of Adverse Experiences on Clinical Outcomes in Individuals with Bipolar Disorder. Brain Sci. 2020;10: https://doi.org/ 10.3390/brainsci10050254.

36. Hawton, K \& Heeringen, K v. The International Handbook of Suicide and Attempted Suicide. Wiley; 2000.

37. Jowett S, Karatzias T, Albert I. Multiple and interpersonal trauma are risk factors for both post-traumatic stress disorder and borderline personality disorder: a systematic review on the traumatic backgrounds and clinical characteristics of comorbid post-traumatic stress disorder/borderline personality disorder groups versus single-disorder groups. Psychol Psychother. 2020;93:621-38. https://doi. org/10.1111/papt.12248.

38. Jokinen J, Nordstrom P. HPA axis hyperactivity and attempted suicide in young adult mood disorder inpatients. J Affect Disord. 2009;116:117-20. https://doi.org/ 10.1016/j.jad.2008.10.015.

39. Guillaume S, Perroud N, Jollant F, Jaussent I, Olié E, Malafosse A, et al. HPA axis genes may modulate the effect of childhood adversities on decision-making in suicide attempters. J Psychiatr Res. 2013;47:259-65. https://doi.org/10.1016/j. jpsychires.2012.10.014.

40. Wilcox HC, Fullerton JM, Glowinski AL, Benke K, Kamali M, Hulvershorn LA, et al. Traumatic stress interacts with bipolar disorder genetic risk to increase risk for 
suicide attempts. J Am Acad Child Adolesc. Psychiatry. 2017;56:1073-80. https:// doi.org/10.1016/j.jaac.2017.09.428.

41. van Heeringen $\mathrm{K}$, Mann JJ. The neurobiology of suicide. Lancet Psychiatry. 2014;1:63-72. https://doi.org/10.1016/S2215-0366(14)70220-2.

42. Furczyk K, Thome J. Adult ADHD and suicide. Atten Defic Hyperact Disord. 2014;6:153-8. https://doi.org/10.1007/s12402-014-0150-1.

43. Lan WH, Bai YM, Hsu JW, Huang KL, Su TP, Li CT, et al. Comorbidity of ADHD and suicide attempts among adolescents and young adults with bipolar disorder: a nationwide longitudinal study. J Affect Disord. 2015;176:171-5. https://doi.org/ 10.1016/j.jad.2015.02.007.

44. Brent DA, Mann JJ. Family genetic studies, suicide, and suicidal behavior. Am J Med Genet Part C, Semin Med Genet. 2005;133C:13-24. https://doi.org/10.1002/ ajmg.c.30042.

45. McCall WV, Black CG. The link between suicide and insomnia: theoretical mechanisms. Curr Psychiatry Rep. 2013;15:389. https://doi.org/10.1007/s11920013-0389-9.

46. Wright KM, Britt TW, Bliese PD, Adler AB, Picchioni D, Moore D. Insomnia as predictor versus outcome of PTSD and depression among Iraq combat veterans. J Clin Psychol. 2011;67:1240-58. https://doi.org/10.1002/jclp.20845.

47. Fornaro M, Orsolini L, Marini S, De Berardis D, Perna G, Valchera A, et al. The prevalence and predictors of bipolar and borderline personality disorders comorbidity: Systematic review and meta-analysis. J Affect Disord. 2016;195:105-18. https://doi.org/10.1016/j.jad.2016.01.040.

\section{ACKNOWLEDGEMENTS}

This study was supported by the American Foundation of Suicide Prevention (AFSP) BSG-1-005-18 (VW and HC); the National Institute of Mental Health, R01MH122412 (HC), R01MH123489 (HC), R01MH123619 (AD), the Clark Tanner Foundation (HC and EM), and the Utah Division of Substance Abuse and Mental Health (HC). Processing of Utah suicide samples was done with assistance from the GCRC M01-RR025764 from the National Center for Research Resources. Genotyping of these samples was done with support from Janssen Research \& Development, LLC to the University of Utah. We thank the University of Utah psychiatry residency research track for their support. Partial support for all datasets within the Utah Population Database was provided by the University of Utah Huntsman Cancer Institute. We thank the staff of the Utah Office of the Medical Examiner for their work in making the collection of samples from suicide deaths possible. Please see the Supplementary materials and methods for full NIMH sample acknowledgement.

\section{COMPETING INTERESTS}

Dr. Qingqin Li is an investigator at Janssen Research, LLC. No other conflicts of interest or financial disclosures are reported by any of the other authors.

\section{ADDITIONAL INFORMATION}

Supplementary information The online version contains supplementary material available at https://doi.org/10.1038/s41398-021-01500-w.

Correspondence and requests for materials should be addressed to E.T.M.

Reprints and permission information is available at http://www.nature.com/ reprints

Publisher's note Springer Nature remains neutral with regard to jurisdictional claims in published maps and institutional affiliations.

(C) Open Access This article is licensed under a Creative Commons Attribution 4.0 International License, which permits use, sharing, adaptation, distribution and reproduction in any medium or format, as long as you give appropriate credit to the original author(s) and the source, provide a link to the Creative Commons license, and indicate if changes were made. The images or other third party material in this article are included in the article's Creative Commons license, unless indicated otherwise in a credit line to the material. If material is not included in the article's Creative Commons license and your intended use is not permitted by statutory regulation or exceeds the permitted use, you will need to obtain permission directly from the copyright holder. To view a copy of this license, visit http://creativecommons. org/licenses/by/4.0/.

(c) The Author(s) 2021 